\title{
Acknowledgment to Reviewers of Societies in 2021
}

\author{
Societies Editorial Office
}

Citation: Societies Editorial Office. Acknowledgment to Reviewers of Societies in 2021. Societies 2022, 12, 22. https://doi.org/10.3390/soc12010022

Published: 9 February 2022

Publisher's Note: MDPI stays neutral with regard to jurisdictional claims in published maps and institutional affiliations.

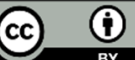

Copyright: () 2022 by the author. Licensee MDPI, Basel, Switzerland. This article is an open access article distributed under the terms and conditions of the Creative Commons Attribution (CC BY) license (https://creativecommons.org/licenses/by/4.0/).

MDPI AG, St. Alban-Anlage 66, 4052 Basel, Switzerland

Rigorous peer-reviews are the basis of high-quality academic publishing. Thanks to the great efforts of our reviewers, Societies was able to maintain its standards for the high quality of its published papers. Thanks to the contribution of our reviewers, in 2021, the median time to first decision was 18 days and the median time to publication was 46 days. The editors would like to extend their gratitude and recognition to the following reviewers for their precious time and dedication, regardless of whether the papers they reviewed were finally published:

Abdul-Rahim Mohammed

Abraham López Vivancos

Adela Badau

Adriana Aubert

Agnieszka Bojanowska

Agnieszka Jaszczak

Agnieszka Wojewodzka-Wiewiorska

Aida Petca

Aïmen Khacharem

Ainhoa Izaguirre

Aje Carlbom

Albin Wagener

Aleksandra Łapko

Alessandra Fermani

Alessandro Sapienza

Alexey Mikhaylov

Alina Betlej

Alton Okinaka

Altovise Ewing

Amélia Marchão

Ana Álvarez Muelas

Ana Isabel Ponce Gea

Ana-Maria Bolborici

Andrea Graus

Andrea López Chao

Andreea Saseanu

Ann Murray

Anna Adamus

Anna Dłużewska

Anna Małgorzata Kamińska

Anna Richiedei

Anna Staszewska

Anna Visvizi
Antonia Pelegrín

Antonio Jesús Molina Fernández

Antonio Luís Martinez-Pujalte

Antonio Luque De La Rosa

Antonio Palacios-Rodríguez

Armando Aliu

Ascanio Sirignano

Asrat Amnie

Atxu Amann

Aurélie Van Hoye

Avril Keating

Ayhan Dolunay

Bahareh Oryani

Bala S. C. Koritala

Barbara Cieślińska

Barbara Ratzenböck

Beata Łubianka

Ben Kisby

Bogusława Urbaniak

Brian Jenkins

Bruno Costa

Bryan R. Brown

Brygida Klemens

Carles Marín Lladó

Carlos David Gómez-Carmona

Carlos De Las Heras-Pedrosa

Carlos Teixeira

Carlton Brick

Carolin Müller

Carson Patterson

Charlie V. Morgan

Charlotte Walker-Said

Cheng Wang 
Cheryl A. Camillo

Chokri Kooli

Christina Laspa

Christine A. James

Christine Marizzi

Christoph Borzikowsky

Christoph Bühren

Christopher Douglas

Christopher W. Mullins

Cinzia Ferranti

Cláudia De Barros Camargo

Claudiu Cicea

Codruta Mare

Conor Mc Guckin

Cristinel Petrișor CONSTANTIN

Czesław Adamiak

Damiano Menin

Dana Mustata

Daniel Muñoz-Sastre

Daniel Silander

Daniel Tower

Daniela Acquadro Maran

Daniela Antonescu

Daniela Cherubini

Daniele Cavaleri

Daniele Ronsivalle

Danielle Nunnery

Danny D. Mann

Dariusz Tworzydło

Dave Bridge

David Alonso González

David Grohmann

David Herbert

David Schultz

Davide Settembre-Blundo

Dawn Stobbart

Despina Chronaki

Devin Heyward

Dikaia Chatziefstathiou

Dimitrios Giomelakis

Dimitris Mourtzis

Dmitry Ruban

Dolores Herrero

Donatella Privitera

Eduardo Lopez

Ehsan Asadollahi

Elena Dimitrova

Elena María Pérez González

Elena Sidorova

Elena-Loreni Baciu
Elisete Diogo

Elspeth McInnes

Emel Thomas

Emilia Herman

Emilio Crisol-Moya

Eric Wiebelhaus-Brahm

Erin Pritchard

Eva Martin-Fuentes

Evanthia Sakellari

Evgenia Anastasiou

F. Javier Del Río

Federico Camerin

Federico Roggio

Fernando Rubén García Hernández

Florina Virlanuta

Francesc Fusté-Forné

Francesca Comunello

Francesco Grimaldi

Francisco Branco

Francisco Mateos Claros

Francisco Rodríguez

Frank Li

Fred Wulczyn

Gabriel López-Martínez

Gallego M. Dolores

George Jennings

George Tsaramirsis

Gianluigi Busico

Ginna Brock

Giovanna Campani

Grigoras Gheorghe

Hala Ibrahim Ghoneim

Hannah Wechkunanukul

Hans-Georg Betz

Helene Diana Connor

Henk Erik Meier

Hocine Bougdah

Hsin-Chi Ko

Hsiu-Ching Chiu

HSIU-CHING KO

Huey-Hong Hsieh

Hui-Chi Li

Hung-Yi Chuang

Hyeon-Cheol Kim

Hyo Sun Jung

Ieva Stončikaitè

Ilan Katz

Ilias Kapareliotis

Inmaculada Méndez Mateo

Ioan Ungurean 


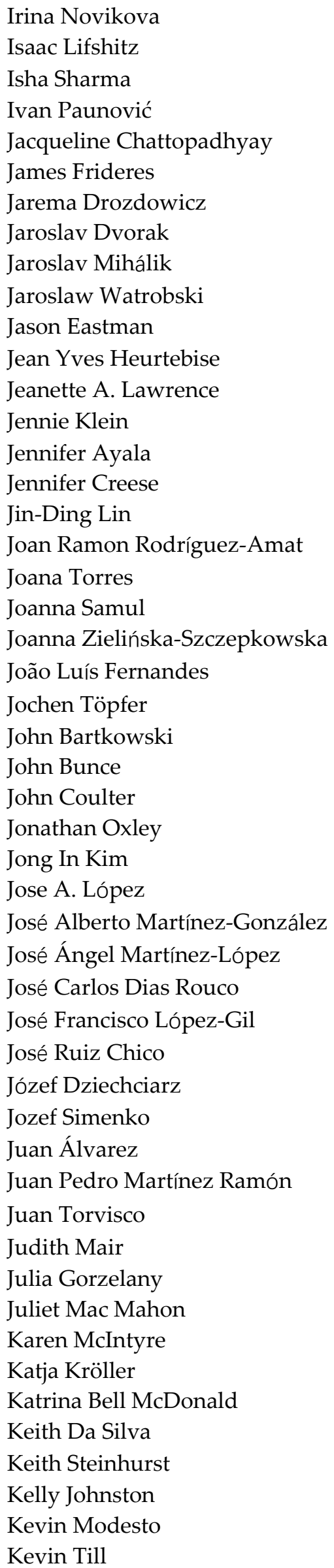

Kristen Ethier

Kristina Matuzeviciute

Krystyna Poznańska

Larry Nackerud

Lars Dzikus

Laura Cervi

Laura Guerrero-Puerta

Laura Louise Nicklin

Lauren Mims

Laurentiu-Gabriel Talaghir

Lavinia Stan

Lázaro Florido-Benítez

Lee Michael Savage

Liběna Tetřevová

Liliana Radu

Loredana Ivan

Lorena Dadić

Louisa J. M. Jansen

Lucian Lupu-Dima

Lucie Corcoran

Lucie Drechselová

Luis A. Saboga-Nunes

Luísa Dias Pereira

Luiza Mesesan Schmitz

Luminita Nicolescu

Magdalena M. Stuss

Magdalena Romera

Maja Mesko

Maja Turnsek

Małgorzata Kisilowska

Malvina N. Skorska

Marc Dreyer

Marco G. Alves

Margareta Gregurović

Margo Turnbull

Maria Angeles Martinez Ruiz

María Del Carmen Olmos Gómez

Maria Grajdian

María José Camacho-Miñano

María-Leticia Meseguer-Santamaría

Marian Simion

Maria-Therese Friehs

Marietta Kiss

Marijana Majdak

Marina M. S. Cabral-Pinto

Marit Kristine Helgesen

Marjeta Šinko

Marta Stojić Mitrović

Martin Hand

Martin Sanchez-Gomez 
Martin Thomas Falk

Mary Moloney

Masoud Esfandiari

Matt Coward-Gibbs

Maurizio Valenti

Max O. Stephenson

Meelis Kitsing

Meline Kevorkian

Melissa C. Osborne

Mert Baştaş

Michael Grothe-Hammer

Michał Bronikowski

Michal Pink

Michał T. Tomczak

Michele Fontefrancesco

Mieczysław P. Boduszyński

Miguel Castro

Mihaela Kardos

Mihai-Stelian Rusu

Mihály Kökény

Mikhail Kozhevnikov

Míriam Díez Bosch

Mita Banerjee

Monica Aureliana Petcu

Monika Parchomiuk

Mostafa Elgendy

Muhammad Chutiyami

Mykolas Simas Poškus

Nader Asnafi

Nadezhda Sivrikova

Natalia Wagner

Nevena Kulic

$\mathrm{Ng}$ Chiew Hong

Nicholas Birns

Nora Repo-Saeed

Norbert Schütte

Nuno De Almeida Alves

Nuria Rodríguez-López

Oana Şerban

Octavian Moldovan

Olga Fragou

Olga Magano

Pablo A. Cantero-Garlito

Panagiotis Kyratsis

Panagiotis Pentaris

Pat Thane

Paul McCafferty

Pavel Krpálek

Pedro A. Martin Cervantes

Pedro Cuesta Valiño
Peter W. Choate

Peter Winwood

Petra Nordqvist

Phillip J. Granberry

Platon Tinios

Po-Fu Lee

Primus M. Tazanu

Puiu Nistoreanu

Qi Jin

Rafael López-Cordero

Rafal Kocylowski

Rafal Soborski

Rail M. Shamionov

Ranjan Datta

Raquel Alarcón

Rasa Pocevičienè

Raul Navarro

Raul Ramos

Reima Suomi

Riccardo Ladini

Richard L. Wolfel

Rinaldo Paar

Roar Eilev Amdam

Robert James Crammond

Rodica Ianole-Calin

Rogobete Silviu

Romina Fucà

Rui Silva

Ryuichi Ohta

Sabrina Lai

Samuel Chng

Sandro Serpa

Santiago Pérez-Nievas

Sara Miccoli

Sara Nunes

Sergio A. Useche

Sergio Calonge-Pascual

Sergio Serra

Shamika Ann Mitchell

Shankar Ghimire

Shirley A. Hill

Shirley Wyver

Shiva Hadi Esfahani

Sibylle Heilbrunn

Simon Darcy

Simona Kuti

Sławomir Gawroński

Sofya Aptekar

Sonja Novak-Zezula

Sophia Moskalenko 
Spyridon Kaltsas

Stefan Bratosin

Steinunn Helga Lárusdóttir

Stephanie Koerner

Steven Kairys

Sunghyup Sean Hyun

Taewoo Nam

Tamás Kovalcsik
Timothy Lenz

Verónica Marín-Díaz

Vsevolod V. Konstantinov

Wai Sum Lee

Walter Schumm

Xosé Somoza-Medina

Zoltan Baracskai 